\title{
Erratum
}

Journal of Microbiology (2019) Vol. 57, No. 11, pp. 959-966

DOI 10.1007/s12275-019-9007-9

\section{Methylobacterium terrae sp. nov., a radiation-resistant bacterium isolated from gamma ray-irradiated soil}

\author{
Jiyoun Kim ${ }^{1}$, Geeta Chhetri ${ }^{1}$, Inhyup Kim ${ }^{1}$, Hyungdong Kim ${ }^{1}$, Myung Kyum Kim ${ }^{2}$, and Taegun Seo ${ }^{1 \star}$ \\ ${ }^{1}$ Department of Life Science, Dongguk University-Seoul, Goyang 10326, Republic of Korea \\ ${ }^{2}$ Department of Bio \& Environmental Technology, College of Natural Science, Seoul Women's University, Seoul 01797, Republic of Korea
}

In the article by Kim et al. published in Journal of Microbiology 2019; 57, 959-966, The NBRC accession number NBRC $112879^{\top}$ on $33^{\text {th }}$ line of $2^{\text {nd }}$ paragraph in the section of 'Description of Methylobacterium terrae sp. nov.' on page 964 should be corrected in NBRC $112873^{\top}$.

The sentence in abstract should have read: The GenBank/EMBL/DDBJ accession numbers for the 16S rRNA gene and genome

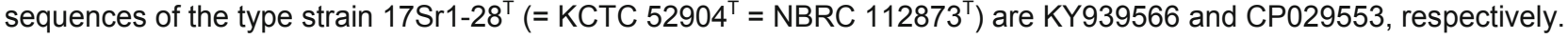

We apologize for any inconvenience that this may have caused. 